\title{
Finger Vein Recognition Algorithm Using Phase Only Correlation
}

\author{
Nurhafizah Mahri*†, Shahrel Azmin Sundi @ Suandi* and Bakhtiar Affendi Rosdi* \\ *Intelligent Biometric Group, School of Electrical and Electronic Engineering \\ Universiti Sains Malaysia, 14300 Nibong Tebal, Malaysia \\ ${ }^{\dagger}$ Faculty of Electrical and Electronic Engineering \\ Universiti Tun Hussein Onn Malaysia, 86400 Parit Raja, Malaysia \\ Email: hafizahm@uthm.edu.my, shahrel@eng.usm.my, eebakhtiar@eng.usm.my
}

\begin{abstract}
In this paper, we propose an algorithm for finger vein recognition with less complexity in the image preprocessing phase, where finger vein pattern extraction is not included at all. In the proposed algorithm, we implement phase-only correlation (POC) function at the matching stage with a very simple preprocessing technique. Experimental evaluation of the proposed algorithm using a set of finger vein images captured from a low cost device have resulting an efficient recognition performance where the equal error rate (EER) was $0.9803 \%$ with a total processing time of $0.6362 s$.
\end{abstract}

\section{INTRODUCTION}

Finger vein is a blood vessel network under finger skin. The network pattern is unique for each individual [1], unaffected by aging, and it is internal, i.e. inside human skin which can always guarantee high security authentication. Nowadays, finger vein has become one of the major interest in biometric research for automated system due to it attributes in high security and reliability. Accordingly, a lot of new devices and technologies which are related to finger vein recognition have emerged in the worldwide market.

Research [2], [3], [4] on finger vein recognition that have been proposed have similarities on the fundamental algorithm which include finger vein pattern extraction in the preprocessing stage. However, when the vein pattern is not clear, pattern extraction might become inconvenient and prone to extract inaccurate vein pattern [5]. To overcome this problem, recently, researchers have developed an efficient matching technique using the phase component of two-dimensional discrete Fourier Transform (2D-DFT) of an image [6]. This matching technique is also known as Phase Only Correlation (POC). It has become a current emerging matching technique in biometric application such as fingerprint [6] and iris [7] recognition, and finger-knuckle-print verification [8]. This matching technique is claimed reliable, robust and doing less job in pattern extraction.

Correspond to the benefit of using finger vein image as a biometric trait and the performance of POC matching technique, this work proposes an efficient finger vein recognition algorithm using POC function. A preprocessing and matching algorithm have been developed using POC function for a set of finger vein images which have been captured using a low cost finger vein image acquisition device. To summarize, our contributions in this paper are (1) a new finger vein recognition algorithm without vein pattern extraction by utilizing POC function, and (2) demonstrate that POC function is applicable to finger vein recognition.

\section{IMAGE ACQUISITION}

The finger vein images that have been used to demonstrate the proposed algorithm are captured by our own low cost prototype device, as shown in Figure 1, which is constructed from a modified image sensor, Logitech V-UAV35 web-cam and illuminated by Osram SFH485 infrared light emitting diodes (IR LED) with wavelength $880 \mathrm{~nm}$. The web-cam is not infrared (IR) sensitive device where it consists an IR blocking filter. Hence, the IR blocking filter is replaced by a negative film to react as an IR pass filter. From [9], it has been proved that negative film can react as IR pass filter where it can transmit about $90 \%$ radiation wavelength of $850 \mathrm{~nm}$.
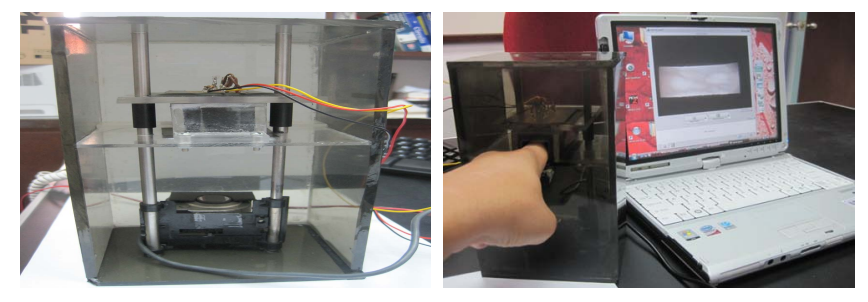

Fig. 1. Finger vein image capturing device.

\section{Phase Only Correlation}

The most significant property of phase only correlation (POC) function is its accuracy in image matching. This section explains the implementation of POC function on finger vein image. The definition of the POC function was explained in detail by [6].

Considering two images $f(m, n)$ and $g(m, n)$, each with $(2 N+1) \times(2 M+1)$ fixed size image where the index range are $m=-M \ldots M$ and $n=-N \ldots N$. Let $F(u, v)$ and $G(u, v)$ denote the 2-dimensional Discrete Fourier Transform (DFT) 
of the two images. $F(u, v)$ is given by

$$
\begin{aligned}
F(u, v) & =\sum_{m=-M}^{M} \sum_{n=-N}^{N} f(m, n) e^{\frac{-2 j u m \pi}{M}} e^{\frac{-2 j v n \pi}{N}} \\
& =A_{F}(u, v) e^{j \theta_{F}(u, v)}
\end{aligned}
$$

where $u=-M \ldots M$ and $v=-N \ldots N . A_{F}(u, v)$ is the amplitude and $\theta_{F}(u, v)$ is the phase. $G(u, v)$ is also defined in the same way. The cross phase spectrum of the two images is given by

$$
R_{F G}(u, v)=\frac{F(u, v) \overline{G(u, v)}}{\mid F(u, v) \overline{G(u, v) \mid}}=e^{j \theta(u, v)}
$$

where $\overline{G(u, v)}$ is the complex conjugate of $G(u, v)$ and $\theta(u, v)$ denotes the phase difference of $\theta_{F}(u, v)-\theta_{G}(u, v)$. The POC function of $r_{f g}(m, n)$ is the 2D inverse DFT (2D IDFT) of $R_{F G}(u, v)$ and is given by

$$
r_{f g}(m, n)=\frac{1}{M N} \sum_{u=-M,}^{M} \sum_{v=-N}^{N} R_{F G}(u, v) \frac{2 j u m \pi}{e^{M}} e^{\frac{2 j v n \pi}{N}}
$$

From [6], if $f(m, n)$ and $g(m, n)$ are two identical images, the POC function is the Kronecker's delta function, $\delta(n, m)$. The score will be degraded if there exist any movement or dissimilarities.

Figure 2 shows three finger vein images and their frequency spectrums. $f_{1}(m, n)$ and $f_{2}(m, n)$ are finger vein images of the same finger, while $g_{1}(m, n)$ is finger vein image of different finger. Table I shows the matching scores of the images using POC function. If two identical finger vein images are matched, the normalized score is equal to one. But if two different images of the same finger are matched, the score is degraded. There are several factors that contribute to this drop such as displacement, translation or rotation of the finger vein image.

From Table I, by using POC function, the matching score of two different images of the same finger is 0.2036. But if two vein images of different finger are matched, there will be a drop in the matching score, in this case the matching score is 0.0917 .

Previous research [6] mentioned about meaningless phase component in high frequency domain. The detail of an image is dominated by low frequency component, where the spectrum power is much higher than the high frequency component. For example, the spectrum of the vein images in Figure 2(d),(e),(f), highlighted the low frequency component that contain the details of the vein image, which is lighter and distributed around the center of the image spectrum. The high frequency component in the finger vein spectrum image is much darker, which mean the high frequency spectrum have a very low power. Hence, by including the non-dominant high frequency phase component during the calculation of crossphase spectrum, the matching score may have less reliability.

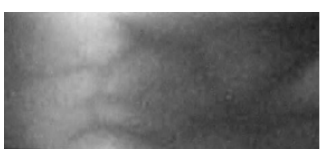

(a)

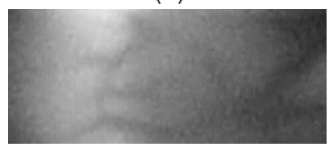

(b)

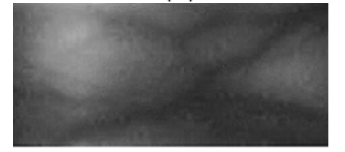

(c)

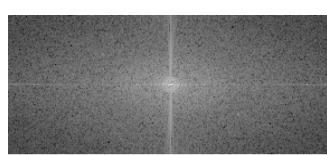

(d)

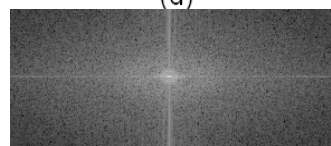

(e)

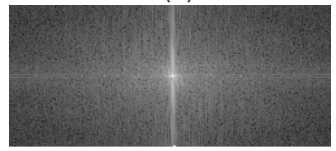

(f)
Fig. 2. Example of finger vein images and their frequency spectrums: (a) and (b) are finger vein images of the same finger $f_{1}(m, n)$ and $f_{2}(m, n)$, respectively, and (c) $g_{1}(m, n)$ is finger vein image of different finger, while (d), (e), and (f) are frequency spectrums of $f_{1}(m, n), f_{2}(m, n)$, and $g_{1}(m, n)$, respectively.

TABLE I

MATCHING SCORES Using POC AND BLPOC FUnCTION

\begin{tabular}{|c|c|c|c|}
\hline Input Image & Registered Image & POC & BLPOC \\
\hline \hline$f_{1}(m, n)$ & $f_{1}(m, n)$ & 1 & 1 \\
\hline$f_{1}(m, n)$ & $f_{2}(m, n)$ & 0.2036 & 0.6791 \\
\hline$f_{1}(m, n)$ & $g_{1}(m, n)$ & 0.0917 & 0.1940 \\
\hline
\end{tabular}

In order to eliminate the meaningless high frequency component, it is excluded in the calculation of cross-phase spectrum. The high frequency component is eliminated by setting a band-limit during calculation of the cross-phase spectrum. The calculation of the cross-phase spectrum only include $q$ of the spectrum size, i.e. $q *(2 N+1) \times q *(2 M+1)$, origin at the correlation peak location, which always at the centre of the frequency spectrum. This method is also known as bandlimited POC (BLPOC) function [6].

The matching scores of the same and different finger vein images using BLPOC function are shown in Table I. For this example, the band-limit or the value of $q$ is set to 0.1. As expected, the score is emphasized and more distinguishable. The effect of using BLPOC function is illustrated in Figure 3, where the correlation peak is increased to a high desirable score. Since determining the optimum band limit is very crucial, an experiment is designed to determine the optimum band limit for this particular finger vein image matching, which is explained in the next section.

\section{Finger Vein Recognition Algorithm}

This section explains the finger vein recognition algorithm using BLPOC function. Figure 4 shows the flow diagram of the proposed finger vein recognition algorithm. The proposed algorithm consists of (i) image normalization, (ii) region of interest (ROI) extraction, (iii) image enhancement using contrast limited adaptive histogram equalization, and (iv) displacement correction and matching score calculation using BLPOC function. 


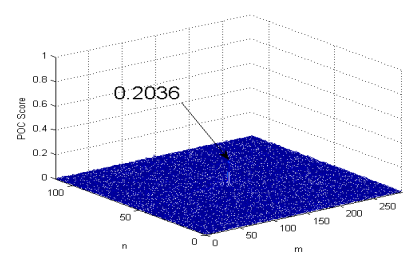

(a)

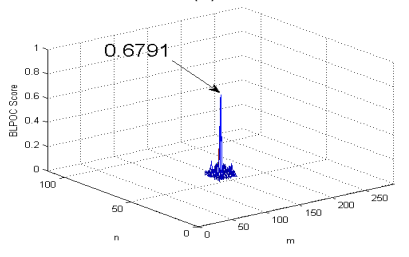

(b)

Fig. 3. (a) POC function and (b) BLPOC function of $f_{1}(m, n)$ and $f_{2}(m, n)$, respectively.

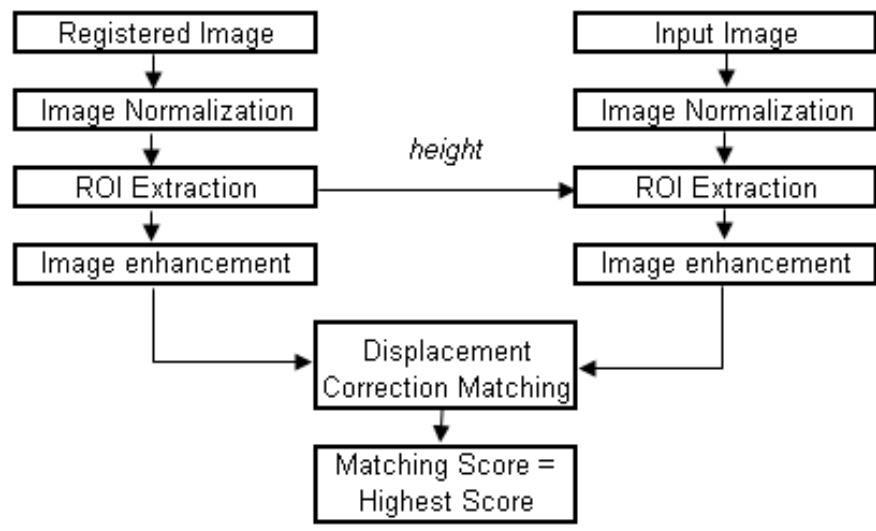

Fig. 4. Flow diagram of the proposed algorithm

\section{A. Image Normalization}

In order to achieve high accuracy finger vein image matching, the original image is normalized into smaller size. This step is simple, the original image size of $640 \times 480$ pixels is reduced to a smaller resolution, $384 \times 288$ pixels, where the scale is 0.6 of the original image size. This is the optimum scaling factor, which is obtained from the experiment of the vein image database for various scaling factor at fix other parameters. Moreover, processing speed is also reduced with the scaling factor.

\section{B. ROI Extraction}

The original image is captured with the black unwanted background. Including the background reduced the accuracy of

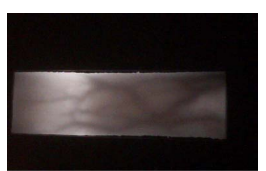

(a)

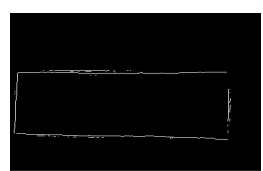

(b)

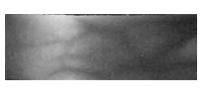

(c)
Fig. 5. (a) Original finger vein image, (b) finger edges, and (c) cropped image.

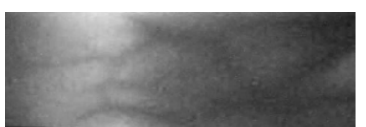

(a)

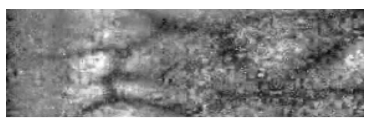

(b)
Fig. 6. (a) Before and (b) after image enhancement.

the matching algorithm using BLPOC function. Therefore, the original image is extracted from the undesired background. A special algorithm is developed to extract the finger vein image from the background.

Three major steps involved in this algorithm. First, edge detection is performed to highlight the finger edge points. There are two major horizontal lines detected representing the finger edges as shown in Figure 5(b). Second, pairs of edge points are determined from each of the two major horizontal lines by scanning the lines horizontally. The most appropriate cropping points are selected from the pairs of edge points, which must satisfy two conditions: (i) the range of the pair of the edge points is between $35 \%$ to $65 \%$ of the image height, and (ii) the pair of the edge points is the widest pair among all pairs. Finally, the image is cropped vertically at the cropping points and horizontally at 5\% from left border and 15\% from right border.

For matching purpose, the size of both registered and input images are preserved to be at the same size. The ROI of input image is depending on the ROI of registered image. From the detected cropping points of the input image, the centre of the cropping points is calculated. The input image is cropped at the same height of the registered image origin from the calculated cropping points centre. Figure 5 shows an original finger vein image, finger edges, and the final cropped image.

\section{Image Enhancement}

This step is to highlight the finger vein network pattern in order to increase the matching accuracy using BLPOC function. In this work, contrast limited adaptive histogram equalization (CLAHE) [10] is used to enhance the image. By using this method, the finger vein network pattern is much clearer and contribute to a higher accuracy matching score. Figure 6 illustrates a finger vein image before and after image enhancement.

\section{Displacement Correction Matching}

Implementing BLPOC function as a matching technique prone to displacement error, and the matching score is reduced due to the displacement of the finger vein image. For our specific device, most of the displacement errors are contribution of horizontal displacement. Therefore no displacement correction is done for vertical and diagonal since the performance of the algorithm is not much affected, and the matching time can be reduced. Due to this matter, four sets of horizontally displaced image are created in advance.

Figure 7 illustrates the registered and input image areas that are included and discarded for horizontal displacement correction analysis. The shadowed areas are the included area 


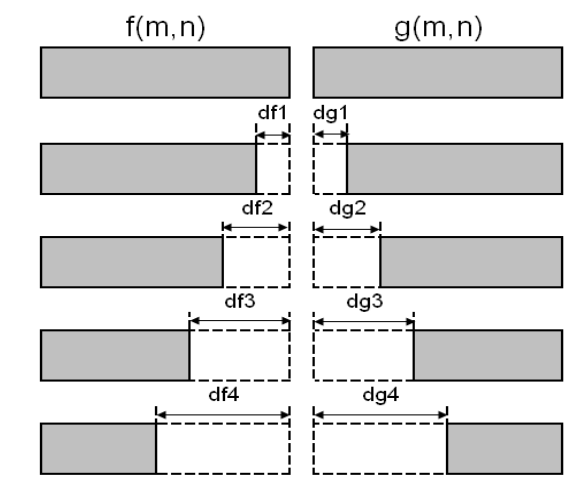

Fig. 7. Four pairs of registered image, $f(m, n)$ and input image, $g(m, n)$ for horizontal displacement correction matching.

and the non-shadowed areas are the discarded area. The width of the discarded areas, registered image $(d f 1, d f 2, d f 3, d f 4)$ and input image $(d g 1, d g 2, d g 3, d g 4)$ are 2 pixels, 4 pixels, 6 pixels and 8 pixels, respectively. In this method, for every set of images, the BLPOC function is calculated between the shadowed areas of the registered and input images. The highest score among the five sets (including the first set with no discarded area) is selected as the final matching score.

\section{EXPERIMENTS AND DISCUSSION}

The finger vein image database that has been used in this project was established using the capturing device that was explained in Section II. The images were collected from 51 male and female volunteers, whom are staffs and students of Universiti Sains Malaysia. The age of the subjects ranged from 21 to 56 years old. Each subject provides 10 images of four fingers, which are left index finger, left middle finger, right index finger and right middle finger. Therefore, there are 51 sets of four fingers with 10 images of each finger. In total, the database contains 2040 images from 204 different fingers. The processing time in this work is evaluated using Matlab Version 7.5.0.324(R2007b) on Intel Dual Core $1.66 \mathrm{GHz}$ processor with 1GB RAM.

A set of experiments have been conducted using the established database in order to evaluate the matching performance of the proposed algorithm. The results of these experiments have produced an optimum parameters for the best matching performance. From [11], they have changed the image size and band-limit of BLPOC function to optimize the performance. Similarly in this work, the same method is applied for the same purpose.

There are three major experiments will be explained in this section, which are (i) experiment to determine the optimum band limit for BLPOC matching algorithm, (ii) experiment to determine the optimum scaling factor and (iii) experiment for displacement correction. The performance of the matching algorithm in this work is evaluated using Equal Error Rate as in [2], [3], [4], [5]. The EER is defined as the error rate when the False Acceptance Rate (FAR) and the False Rejection Rate
TABLE II

EXPERIMENTAL RESULTS ON BAND-LIMIT RATIO, $q$, WITH IMAGE SCALING FACTOR 0.6.

\begin{tabular}{|c|c|c|c|}
\hline $\mathbf{q}$ & EER\% & Threshold & Times (s) \\
\hline \hline 0.06 & 2.4950 & 0.360 & 0.1913 \\
\hline 0.08 & 1.7400 & 0.290 & 0.1976 \\
\hline 0.10 & 1.4435 & 0.250 & 0.3249 \\
\hline 0.12 & 1.5060 & 0.210 & 0.1966 \\
\hline 0.15 & 1.9225 & 0.180 & 0.2008 \\
\hline 0.20 & 4.8150 & 0.160 & 0.1985 \\
\hline 0.25 & 7.6855 & 0.150 & 0.2018 \\
\hline 0.30 & 10.130 & 0.140 & 0.3196 \\
\hline
\end{tabular}

TABLE III

EXPERIMENTAL RESULTS ON IMAGE SCALING FACTOR WITH BAND-LIMIT RATIO $q=0.1$.

\begin{tabular}{|c|c|c|c|}
\hline Scale & EER\% & Threshold & Times (s) \\
\hline \hline 0.4 & 2.4510 & 0.340 & 0.2297 \\
\hline 0.5 & 1.8110 & 0.280 & 0.2606 \\
\hline 0.6 & 1.4435 & 0.250 & 0.3249 \\
\hline 0.7 & 1.5308 & 0.215 & 0.4908 \\
\hline 0.8 & 1.7755 & 0.310 & 0.4934 \\
\hline
\end{tabular}

(FRR) are equal.

\section{A. Band-Limit Optimization}

Referring to [11], the optimization of setting the band-limit can be achieved by varying the ratio of the band-limit to the image size. In this experiment, the scale is fixed to 0.6 of the image size while the band limit is varied according to the ratio of the band-limit to the image size (considering the image is already cropped from the background). For example if the image size is $M \times N$ pixels, it will be reduced to $0.6 * M \times$ $0.6 * N$ pixels, and the band limit is set to $q$ of the image size. If $q=0.10$, the band limit is $0.10 *(0.6 * M) \times 0.10 *(0.6 * N)$. Table II shows the experimental results of varying the bandlimit ratio, q. From Table II, the optimum band-limit ratio is 0.10 where the EER is $1.4435 \%$ at threshold level 0.250 . Nevertheless, the processing time of the matching process is not much affected by varying the ratio as in [11], where in this experiment the average processing time is $0.2285 \mathrm{~s}$.

\section{B. Scaling Factor Optimization}

As well as the band limit ratio, the scaling factor might contribute to the performance as described in [11]. Due to that, the finger vein image database is experimented with various scaling factor with the band-limit ratio, $q$ is set to 0.10 . Table III shows the experimental results of varying the image scaling factor from 0.4 to 0.8. From Table III, it can be observed that if the scale is too small, the processing time is reduced but the performance is dropped. On the other hand, if the scale is too large, the processing time is increased as well as the performance is dropped. The optimum scaling factor shown in Table III is 0.6 of the original image, where the EER is $1.4435 \%$ at threshold level 0.250 with processing time $0.3249 \mathrm{~s}$. 


\section{Displacement Correction}

In this experiment, the database is tested with three different types of displacement at optimum parameters, where the bandlimit ratio, $q=0.1$ and the image scaling factor is 0.6. The details of the experiments are described in Table IV. Type A is without any displacement correction. Type B consists of three displacement corrections, which are horizontal, vertical, and diagonal displacement, while Type $\mathrm{C}$ is only limited to horizontal displacement correction. From Table IV, the performance is improved with displacement correction, but it has to compensate with the processing time. The processing time is increased significantly if all the displacement corrections are performed. But if the displacement correction is only taking into account the horizontal one (type $\mathrm{C}$ ), the processing time is reduced at about twice of type $\mathrm{B}$, and the performance is still compatible with type $\mathrm{B}$, where all the three displacement corrections are performed. It can be concluded that, the image database that we have captured using our designed device is much dis-aligned by horizontal displacement.

TABLE IV

EXPERIMENTAL RESULTS ON DISPLACEMENT CORRECTION WITH OPTIMUM PARAMETERS (BAND-LIMIT RATIO $q=0.1$ AND IMAGE SCALING FACTOR $=0.6)$.

\begin{tabular}{|c|c|c|c|c|}
\hline Type & Displacement & EER\% & Threshold & Times (s) \\
\hline \hline A & None & 1.4435 & 0.250 & 0.3249 \\
\hline B & Horizontal,Vertical,Diagonal & 0.7598 & 0.290 & 1.0609 \\
\hline C & Horizontal & 0.9803 & 0.265 & 0.6362 \\
\hline
\end{tabular}

Figure 8 illustrates the genuine and impostor matching distribution of the proposed method. The matching distribution shows that the genuine and impostor matching scores can be distinguished at threshold level 0.265. Similarly in Figure 9, the EER plot of our proposed method is $0.9803 \%$ at threshold level 0.265 . As mentioned earlier, the database consists of 51 subjects, four fingers for each subject, and 10 images for each finger. This is equivalent to 204 classes $(51 \times 4)$ with 10 images for each class. Matching score for each test depends on the registered image. Different registered image will result different matching score. Therefore, in the genuine tests for each class, each image in the class will be choosed as an input image, and will be matched with all registered images in the same class. This will make the number of genuine tests for each class is $90\left(2 \times{ }_{10} C_{2}\right)$. Hence, for 204 classes, the number of genuine tests is $18360(204 \times 90)$. The number of impostor tests can reach to $2061420\left(\left(2 \times{ }_{2040} C_{2}\right)-18360\right)$. However, for this work, the number of impostor tests is set to follow the number of genuine tests which is 18360 .

\section{CONCLUSION}

A novel simple algorithm for finger vein recognition is proposed. The complexity of preprocessing stage using vein pattern extraction method is eliminated with compatible matching performance. In the future, we would like to demonstrate the proposed algorithm with a better quality finger vein image with minimize preprocessing steps and only emphasize on the advantage of BLPOC function.

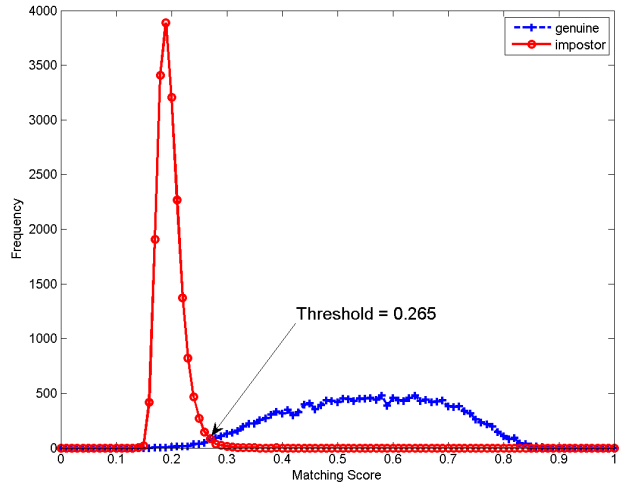

Fig. 8. Matching distribution for genuine and impostor with optimum parameters $(q=0.1$, image scaling factor $=0.6$, and horizontal displacement correction).

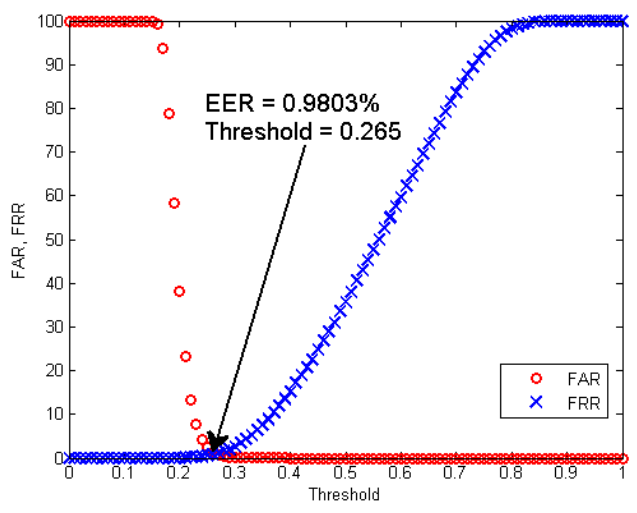

Fig. 9. Matching performance with optimum parameters $(q=0.1$, image scaling factor $=0.6$, and horizontal displacement correction).

\section{ACKNOWLEDGEMENT}

This work is supported by Universiti Sains Malaysia Short Term Grant No. 6039021.

\section{REFERENCES}

[1] L. Xueyen and G. Shuxu. Pattern Recognition, chapter 23: The Fourth Biometric - Vein Recognition, pages 537-546. IN-TECH, November 2008.

[2] N. Miura, A. Nagasaka, and T. Miyatake. Feature extraction of finger vein patterns based on repeated line tracking and its application to personal identification. Machine Vision and Applications, Vol. 15(4):pages 194203, October 2004.

[3] D. Mulyono and H. S. Jinn. A study of finger vein biometric for personal identification. Biometrics and Security Technologies, ISBAST 2008, pages 1-8, June 2008.

[4] Z. B. Zhang, D. Y. Wu, S. L. Ma, and J. Ma. Multi scale feature extraction of finger vein patterns based on curvelets and local interconnection structure neural network. Proceeding of 18th International Conference on Pattern Recognition (ICPR) 2006., pages 145-148, September 2006.

[5] E. C. Lee, H. C. Lee, and K. R. Park. Finger vein recognition using minutia-based alignment and local binary pattern-based feature extraction. International Journal of Imaging Systems and Technology, Vol. 19(3):pages 179-186, August 2009.

[6] K. Ito, H. Nakajima, K. Kobayashi, T. Aoki, and T. Higuchi. A fingerprint matching algorithm using phase-only correlation. IEICE Trans. Fundamentals, Vol. E87-A(3):pages 682-691, March 2004. 
[7] K. Miyazawa, K. Ito, T. Aoki, K. Kobayashi, and H. Nakajima. An effective approach for iris recognition using phase-based image matching. IEEE Transaction On Pattern Analysis And Machine Intelligence, Vol. 30(10):pages 1741-1756, October 2008

[8] L. Zhang, L. Zhang, and D. Zhang. Finger-knuckle-print verification based on band-limited phase-only correlation. Proceedings of the 13th International Conference on Computer Analysis of Images and Patterns, pages 141-148, 2009.

[9] http://www.northcountryradio.com/articles/irfltr.htm.

[10] R. C. Gonzalez, R. E. Woods, and S. L. Eddins. Digital Image Processing Using Matlab. Pearson, 2004.

[11] K. Ito, T. Aoki, H. Nakajima, K. Kobayashi, and T. Higuchi. A palmprint recognition algorithm using phase-only correlation. IEICE Trans. Fundamentals, Vol. E91-A(4):pages 1023-1030, April 2008. 